\title{
ON THIRD-ORDER JACOBSTHAL POLYNOMIALS AND THEIR PROPERTIES
}

\author{
GAMALIEL CERDA-MORALES
}

Received 11 February, 2020

\begin{abstract}
Third-order Jacobsthal polynomial sequence is defined in this study. Some properties involving this polynomial, including the Binet-style formula and the generating function are also presented. Furthermore, we present the modified third-order Jacobsthal polynomials, and derive adaptations for some well-known identities of third-order Jacobsthal and modified third-order Jacobsthal numbers.
\end{abstract}

2010 Mathematics Subject Classification: 11B37; 11B39; 11B83

Keywords: recurrence relation, modified third-order Jacobsthal numbers, third-order Jacobsthal numbers

\section{INTRODUCTION}

The Jacobsthal numbers have many interesting properties and applications in many fields of science (see, [1]). The Jacobsthal numbers $\left(J_{n}\right)_{n \geq 0}$ are defined by the recurrence relation

$$
J_{0}=0, J_{1}=1, \quad J_{n+2}=J_{n+1}+2 J_{n}, n \geq 0 .
$$

Another important sequence is the Jacobsthal-Lucas sequence. This sequence is defined by the recurrence relation $j_{n+2}=j_{n+1}+2 j_{n}$, where $j_{0}=2$ and $j_{1}=1$.

In Cook and Bacon's work [5] the Jacobsthal recurrence relation is extended to higher order recurrence relations and the basic list of identities provided by A. F. Horadam [9] is expanded and extended to several identities for some of the higher order cases. In fact, the third-order Jacobsthal numbers, $\left\{J_{n}^{(3)}\right\}_{n \geq 0}$, and third-order Jacobsthal-Lucas numbers, $\left\{j_{n}^{(3)}\right\}_{n \geq 0}$, are defined by

$$
J_{n+3}^{(3)}=J_{n+2}^{(3)}+J_{n+1}^{(3)}+2 J_{n}^{(3)}, J_{0}^{(3)}=0, J_{1}^{(3)}=J_{2}^{(3)}=1, n \geq 0,
$$

and

$$
j_{n+3}^{(3)}=j_{n+2}^{(3)}+j_{n+1}^{(3)}+2 j_{n}^{(3)}, j_{0}^{(3)}=2, j_{1}^{(3)}=1, j_{2}^{(3)}=5, n \geq 0,
$$

respectively.

Some of the following properties given for third-order Jacobsthal numbers and third-order Jacobsthal-Lucas numbers are used in this paper (for more details, see 
[2-5]). Note that Eqs. (1.7) and (1.11) have been corrected in [3], since they have been wrongly described in [5]. Then, we have

$$
\begin{gathered}
3 J_{n}^{(3)}+j_{n}^{(3)}=2^{n+1}, \\
j_{n}^{(3)}-3 J_{n}^{(3)}=2 j_{n-3}^{(3)}, n \geq 3, \\
J_{n+2}^{(3)}-4 J_{n}^{(3)}=\left\{\begin{array}{ccc}
-2 & \text { if } & n \equiv 1(\bmod 3) \\
1 & \text { if } & n \neq 1(\bmod 3)
\end{array},\right. \\
j_{n+1}^{(3)}+j_{n}^{(3)}=3 J_{n+2}^{(3)}, \\
j_{n}^{(3)}-J_{n+2}^{(3)}=\left\{\begin{array}{ccc}
1 & \text { if } & n \equiv 0(\bmod 3) \\
-1 & \text { if } & n \equiv 1(\bmod 3) \\
0 & \text { if } & n \equiv 2(\bmod 3)
\end{array},\right. \\
j_{n-3}^{(3)}+3 J_{n}^{(3)} j_{n}^{(3)}=4^{n}, \\
\sum_{k=0}^{n} J_{k}^{(3)}=\left\{\begin{array}{ccc}
J_{n+1}^{(3)} & \text { if } & n \neq 0 \\
J_{n+1}^{(3)}-1 & \text { if } & n \equiv 0(\bmod 3)
\end{array}\right)
\end{gathered}
$$

and

$$
\left(j_{n}^{(3)}\right)^{2}-9\left(J_{n}^{(3)}\right)^{2}=2^{n+2} j_{n-3}^{(3)}, n \geq 3 .
$$

Using standard techniques for solving recurrence relations, the auxiliary equation, and its roots are given by

$$
x^{3}-x^{2}-x-2=0 ; x=2, \text { and } x=\frac{-1 \pm i \sqrt{3}}{2} .
$$

Note that the latter two are the complex conjugate cube roots of unity. Call them $\omega_{1}$ and $\omega_{2}$, respectively. Thus the Binet formulas can be written as

$$
J_{n}^{(3)}=\frac{2}{7} 2^{n}-\left(\frac{3+2 i \sqrt{3}}{21}\right) \omega_{1}^{n}-\left(\frac{3-2 i \sqrt{3}}{21}\right) \omega_{2}^{n}
$$

and

$$
j_{n}^{(3)}=\frac{8}{7} 2^{n}+\left(\frac{3+2 i \sqrt{3}}{7}\right) \omega_{1}^{n}+\left(\frac{3-2 i \sqrt{3}}{7}\right) \omega_{2}^{n},
$$

respectively. Now, we use the notation

$$
Z_{n}=\frac{A \omega_{1}^{n}-B \omega_{2}^{n}}{\omega_{1}-\omega_{2}}=\left\{\begin{array}{cll}
2 & \text { if } & n \equiv 0(\bmod 3) \\
-3 & \text { if } & n \equiv 1(\bmod 3) \\
1 & \text { if } & n \equiv 2(\bmod 3)
\end{array},\right.
$$

where $A=-3-2 \omega_{2}$ and $B=-3-2 \omega_{1}$. Furthermore, note that for all $n \geq 0$ we have

$$
Z_{n+2}=-Z_{n+1}-Z_{n}, \quad Z_{0}=2, Z_{1}=-3 .
$$


From the Binet formulas (1.12), (1.13) and Eq. (1.14), we have

$$
J_{n}^{(3)}=\frac{1}{7}\left(2^{n+1}-Z_{n}\right) \text { and } j_{n}^{(3)}=\frac{1}{7}\left(2^{n+3}+3 Z_{n}\right) .
$$

A systematic investigation of the incomplete generalized Jacobsthal numbers and the incomplete generalized Jacobsthal-Lucas numbers was featured in [6]. In [7], Djordjević and Srivastava introduced the generalized incomplete Fibonacci polynomials and the generalized incomplete Lucas polynomials. In [8], the authors investigated some properties and relations involving generalizations of the Fibonacci numbers. In [10], Raina and Srivastava investigated the a new class of numbers associated with the Lucas numbers. Moreover they gave several interesting properties of these numbers.

In this paper, we introduce the third-order Jacobsthal polynomials and we give some properties, including the Binet-style formula and the generating functions for these sequences. Some identities involving these polynomials are also provided.

\section{THE THIRD-ORDER JACOBSTHAL POLYNOMIAL, BINET'S FORMULA AND THE GENERATING FUNCTION}

The principal goals of this section will be to define the third-order Jacobsthal polynomial and to present some elementary results involving it.

For any variable quantity $x$ such that $x^{3} \neq 1$. We define the third-order Jacobsthal polynomial, denoted by $\left\{J_{n}^{(3)}(x)\right\}_{n \geq 0}$. This sequence is defined recursively by

$$
J_{n+3}^{(3)}(x)=(x-1) J_{n+2}^{(3)}(x)+(x-1) J_{n+1}^{(3)}(x)+x J_{n}^{(3)}(x), n \geq 0,
$$

with initial conditions $J_{0}^{(3)}(x)=0, J_{1}^{(3)}(x)=1$ and $J_{2}^{(3)}(x)=x-1$.

In order to find the generating function for the third-order Jacobsthal polynomial, we shall write the sequence as a power series where each term of the sequence correspond to coefficients of the series. As a consequence of the definition of generating function of a sequence, the generating function associated to $\left\{J_{n}^{(3)}(x)\right\}_{n \geq 0}$, denoted by $\{j(t)\}$, is defined by

$$
j(t)=\sum_{n \geq 0} J_{n}^{(3)}(x) t^{n}
$$

Consequently, we obtain the following result:

Theorem 1. The generating function for the third-order Jacobsthal polynomials $\left\{J_{n}^{(3)}(x)\right\}_{n \geq 0}$ is $j(t)=\frac{t}{1-(x-1) t-(x-1) t^{2}-x t^{3}}$.

Proof. Using the definition of generating function, we have

$$
j(t)=J_{0}^{(3)}(x)+J_{1}^{(3)}(x) t+J_{2}^{(3)}(x) t^{2}+\cdots+J_{n}^{(3)}(x) t^{n}+\cdots .
$$

Multiplying both sides of this identity by $-(x-1) t,-(x-1) t^{2}$ and by $-x t^{3}$, and then from Eq. (2.1), we have 


$$
\begin{aligned}
& \left(1-(x-1) t-(x-1) t^{2}-x t^{3}\right) j(t) \\
& =J_{0}^{(3)}(x)+\left(J_{1}^{(3)}(x)-(x-1) J_{0}^{(3)}(x)\right) t+\left(J_{2}^{(3)}(x)-(x-1) J_{1}^{(3)}-(x-1) J_{0}^{(3)}(x)\right) t^{2}
\end{aligned}
$$

and the result follows.

The following result gives the Binet-style formula for $J_{n}^{(3)}(x)$.

Theorem 2. For $n \geq 0$, we have

$$
J_{n}^{(3)}(x)=\frac{x^{n+1}}{x^{2}+x+1}-\frac{\omega_{1}^{n+1}}{\left(x-\omega_{1}\right)\left(\omega_{1}-\omega_{2}\right)}+\frac{\omega_{2}^{n+1}}{\left(x-\omega_{2}\right)\left(\omega_{1}-\omega_{2}\right)},
$$

where $\omega_{1}, \omega_{2}$ are the roots of the characteristic equation associated with the respective recurrence relations $\lambda^{2}+\lambda+1=0$.

Proof. Since the characteristic equation has three distinct roots, the sequence $J_{n}^{(3)}(x)=a(x) x^{n}+b(x) \omega_{1}^{n}+c(x) \omega_{2}^{n}$ is the solution of the Eq. (2.1). Considering $n=0,1,2$ in this identity and solving this system of linear equations, we obtain a unique value for $a(x), b(x)$ and $c(x)$, which are, in this case, $\left(x^{2}+x+1\right) a(x)=x$, $\left(x-\omega_{1}\right)\left(\omega_{1}-\omega_{2}\right) b(x)=-\omega_{1}$ and $\left(x-\omega_{2}\right)\left(\omega_{1}-\omega_{2}\right) c(x)=\omega_{2}$. So, using these values in the expression of $J_{n}^{(3)}(x)$ stated before, we get the required result.

We define the modified third-order Jacobsthal polynomial sequence, denoted by $\left\{K_{n}^{(3)}(x)\right\}_{n \geq 0}$. This sequence is defined recursively by

$$
K_{n+3}^{(3)}(x)=(x-1) K_{n+2}^{(3)}(x)+(x-1) K_{n+1}^{(3)}(x)+x K_{n}^{(3)}(x),
$$

with initial conditions $K_{0}^{(3)}(x)=3, K_{1}^{(3)}(x)=x-1$ and $K_{2}^{(3)}(x)=x^{2}-1$.

We give their versions for the third-order Jacobsthal and modified third-order Jacobsthal polynomials.

For simplicity of notation, let

$$
\begin{aligned}
Z_{n}(x) & =\frac{1}{\omega_{1}-\omega_{2}}\left(\left(x-\omega_{2}\right) \omega_{1}^{n+1}-\left(x-\omega_{1}\right) \omega_{2}^{n+1}\right), \\
Y_{n} & =\omega_{1}^{n}+\omega_{2}^{n} .
\end{aligned}
$$

Then, we can write

$$
J_{n}^{(3)}(x)=\frac{1}{x^{2}+x+1}\left(x^{n+1}-Z_{n}(x)\right)
$$

and

$$
K_{n}^{(3)}(x)=x^{n}+Y_{n}
$$

Then, $Z_{n}(x)=-Z_{n-1}(x)-Z_{n-2}(x), Z_{0}(x)=x$ and $Z_{1}(x)=-(x+1)$.

Furthermore, we easily obtain the identities stated in the following result: 
Proposition 1. For a natural number $n$ and $m$, if $J_{n}^{(3)}(x)$ and $K_{n}^{(3)}(x)$ are, respectively, the n-th third-order Jacobsthal and modified third-order Jacobsthal polynomials, then the following identities are true:

$$
\begin{aligned}
& K_{n}^{(3)}(x)=(x-1) J_{n}^{(3)}(x)+2(x-1) J_{n-1}^{(3)}(x)+3 x J_{n-2}^{(3)}(x), n \geq 2, \\
& J_{n}^{(3)}(x) J_{m}^{(3)}(x)+J_{n+1}^{(3)}(x) J_{m+1}^{(3)}(x)+J_{n+2}^{(3)}(x) J_{m+2}^{(3)}(x) \\
& =\frac{1}{\left(x^{2}+x+1\right)^{2}}\left\{\begin{array}{c}
\left(1+x^{2}+x^{4}\right) \cdot x^{n+m+2} \\
-x^{n+1}\left(\left(1-x^{2}\right) Z_{m}(x)+x(1-x) Z_{m+1}(x)\right) \\
-x^{m+1}\left(\left(1-x^{2}\right) Z_{n}(x)+x(1-x) Z_{n+1}(x)\right) \\
+\left(x^{2}+x+1\right)\left(\omega_{1}^{n} \omega_{2}^{m}+\omega_{1}^{m} \omega_{2}^{n}\right)
\end{array}\right\}, \\
& \left.\left(J_{n}^{(3)}(x)\right)^{2}+\left(J_{n+1}^{(3)}(x)\right)^{2}+\left(\begin{array}{c}
(3) \\
n+2
\end{array}\right)\right)^{2} \\
& \left(1+x^{2}+x^{4}\right) \cdot x^{2 n+2} \\
& =\frac{1}{\left(x^{2}+x+1\right)^{2}}\left\{\begin{array}{c}
-2 x^{n+1}\left(\left(1-x^{2}\right) Z_{n}(x)+x(1-x) Z_{n+1}(x)\right) \\
+2\left(x^{2}+x+1\right)
\end{array}\right\},
\end{aligned}
$$

and $Z_{n}(x)$ as in $E q$. (2.4).

Proof. (2.5): To prove Eq. (2.5), we use induction on $n$. Let $n=2$, we get

$$
\begin{aligned}
(x-1) J_{2}^{(3)}(x)+2(x-1) J_{1}^{(3)}(x)+3 x J_{0}^{(3)}(x) & =(x-1)(x-1)+2(x-1) \\
& =x^{2}-1 \\
& =K_{2}^{(3)}(x) .
\end{aligned}
$$

Let us assume that $K_{m}^{(3)}(x)=(x-1) J_{m}^{(3)}(x)+2(x-1) J_{m-1}^{(3)}(x)+3 x J_{m-2}^{(3)}(x)$ is true for all values $m$ less than or equal $n \geq 2$. Then,

$$
\begin{aligned}
K_{m+1}^{(3)}(x)= & (x-1) K_{m}^{(3)}(x)+(x-1) K_{m-1}^{(3)}(x)+x K_{m-2}^{(3)}(x) \\
= & (x-1)\left((x-1) J_{m}^{(3)}(x)+2(x-1) J_{m-1}^{(3)}(x)+3 x J_{m-2}^{(3)}(x)\right) \\
& +(x-1)\left((x-1) J_{m-1}^{(3)}(x)+2(x-1) J_{m-2}^{(3)}(x)+3 x J_{m-3}^{(3)}(x)\right) \\
& +x\left((x-1) J_{m-2}^{(3)}(x)+2(x-1) J_{m-3}^{(3)}(x)+3 x J_{m-4}^{(3)}(x)\right) \\
= & (x-1) J_{m+1}^{(3)}(x)+2(x-1) J_{m}^{(3)}(x)+3 x J_{m-1}^{(3)}(x) .
\end{aligned}
$$

(2.6): Using the Binet formula of $J_{n}^{(3)}(x)$ in Theorem 2, we have

$$
\begin{aligned}
J_{n}^{(3)}(x) J_{m}^{(3)}(x)+J_{n+1}^{(3)}(x) J_{m+1}^{(3)}(x)+J_{n+2}^{(3)}(x) J_{m+2}^{(3)}(x) \\
=\frac{1}{\left(x^{2}+x+1\right)^{2}}\left\{\begin{array}{c}
\left(x^{n+1}-Z_{n}(x)\right)\left(x^{m+1}-Z_{m}(x)\right) \\
+\left(x^{n+2}-Z_{n+1}(x)\right)\left(x^{m+2}-Z_{m+1}(x)\right) \\
+\left(x^{n+3}-Z_{n+2}(x)\right)\left(x^{m+3}-Z_{m+2}(x)\right)
\end{array}\right\} .
\end{aligned}
$$


Then, we obtain

$$
\begin{aligned}
& J_{n}^{(3)}(x) J_{m}^{(3)}(x)+J_{n+1}^{(3)}(x) J_{m+1}^{(3)}(x)+J_{n+2}^{(3)}(x) J_{m+2}^{(3)}(x) \\
& =\frac{1}{\left(x^{2}+x+1\right)^{2}}\left\{\begin{array}{c}
\left(1+x^{2}+x^{4}\right) \cdot x^{n+m+2} \\
-x^{n+1}\left(Z_{m}(x)+x Z_{m+1}(x)+x^{2} Z_{m+2}(x)\right) \\
-x^{m+1}\left(Z_{n}(x)+x Z_{n+1}(x)+x^{2} Z_{n+2}(x)\right) \\
+Z_{n}(x) Z_{m}(x)+Z_{n+1}(x) Z_{m+1}(x)+Z_{n+2}(x) Z_{m+2}(x)
\end{array}\right\} \\
& =\frac{1}{\left(x^{2}+x+1\right)^{2}}\left\{\begin{array}{c}
\left(1+x^{2}+x^{4}\right) \cdot x^{n+m+2} \\
-x^{n+1}\left(\left(1-x^{2}\right) Z_{m}(x)+x(1-x) Z_{m+1}(x)\right) \\
-x^{m+1}\left(\left(1-x^{2}\right) Z_{n}(x)+x(1-x) Z_{n+1}(x)\right) \\
+\left(x^{2}+x+1\right)\left(\omega_{1}^{n} \omega_{2}^{m}+\omega_{1}^{m} \omega_{2}^{n}\right)
\end{array}\right\} .
\end{aligned}
$$

Then, we obtain the Eq. (2.7) if $m=n$ in Eq. (2.6).

\section{SOME IDENTITIES INVOLVING THIS TYPE OF POLYNOMIALS}

In this section, we state some identities related with these type of third-order polynomials. As a consequence of the Binet formula of Theorem 2, we get for this sequence the following interesting identities.

Proposition 2 (Catalan-like identity). For a natural numbers $n$, $s$, with $n \geq s$, if $J_{n}^{(3)}(x)$ is the $n$-th third-order Jacobsthal polynomials, then the following identity

$$
\begin{aligned}
& J_{n+s}^{(3)}(x) J_{n-s}^{(3)}(x)-\left(J_{n}^{(3)}(x)\right)^{2} \\
& =\frac{1}{\left(x^{2}+x+1\right)^{2}}\left\{\begin{array}{c}
x^{n+1}\left(x^{s}-x^{-s}\right) X_{s} Z_{n+1}(x) \\
-x^{n+1}\left(2+x^{s} X_{s+1}-x^{-s} X_{s-1}\right) Z_{n}(x) \\
-\left(x^{2}+x+1\right) X_{s}^{2}
\end{array}\right\}
\end{aligned}
$$

is true, where $Z_{n}(x)$ as in Eq. (2.4), $X_{n}=\frac{\omega_{1}^{n}-\omega_{2}^{n}}{\omega_{1}-\omega_{2}}$ and $\omega_{1}, \omega_{2}$ are the roots of the characteristic equation associated with the recurrence relation $x^{2}+x+1=0$.

Proof. Using the Eq. (2.4) and the Binet formula of $J_{n}^{(3)}(x)$ in Theorem 2, we have

$$
\begin{aligned}
& J_{n+s}^{(3)}(x) J_{n-s}^{(3)}(x)-\left(J_{n}^{(3)}(x)\right)^{2} \\
& =\frac{1}{\left(x^{2}+x+1\right)^{2}}\left\{\begin{array}{c}
\left(x^{n+s+1}-Z_{n+s}(x)\right)\left(x^{n-s+1}-Z_{n-s}(x)\right) \\
-\left(x^{n+1}-Z_{n}(x)\right)^{2}
\end{array}\right\} \\
& =\frac{1}{\left(x^{2}+x+1\right)^{2}}\left\{\begin{array}{c}
-x^{n+1}\left(x^{s} Z_{n-s}(x)+x^{-s} Z_{n+s}(x)-2 Z_{n}(x)\right) \\
+Z_{n+s}(x) Z_{n-s}(x)-\left(Z_{n}(x)\right)^{2}
\end{array}\right\} .
\end{aligned}
$$

Using the following identity for the sequence $Z_{n}(x)$ :

$$
Z_{n+s}(x)=X_{s} Z_{n+1}(x)-X_{s-1} Z_{n}(x),
$$


where $X_{s}=\frac{\omega_{1}^{s}-\omega_{2}^{s}}{\omega_{1}-\omega_{2}}$ and $X_{-s}=-X_{s}$. Then, we obtain

$$
\begin{aligned}
& J_{n+s}^{(3)}(x) J_{n-s}^{(3)}(x)-\left(J_{n}^{(3)}(x)\right)^{2} \\
& =\frac{1}{\left(x^{2}+x+1\right)^{2}}\left\{\begin{array}{c}
x^{n+1}\left(x^{s}-x^{-s}\right) X_{s} Z_{n+1}(x) \\
-x^{n+1}\left(x^{s} X_{s+1}-x^{-s} X_{s-1}-2\right) Z_{n}(x) \\
-\left(x^{2}+x+1\right) X_{s}^{2}
\end{array}\right\} .
\end{aligned}
$$

Hence the result holds.

Note that for $s=1$ in the Catalan-like identity obtained, we get the Cassini-like identity for the third-order Jacobsthal polynomial. Furthermore, for $s=1$, the identity stated in Proposition 2, yields

$$
\begin{aligned}
& J_{n+1}^{(3)}(x) J_{n-1}^{(3)}(x)-\left(J_{n}^{(3)}(x)\right)^{2} \\
& =\frac{1}{\left(x^{2}+x+1\right)^{2}}\left\{\begin{array}{c}
x^{n+1}\left(x^{1}-x^{-1}\right) X_{1} Z_{n+1}(x) \\
-x^{n+1}\left(x^{1} X_{1+1}-x^{-1} X_{1-1}-2\right) Z_{n}(x) \\
-\left(x^{2}+x+1\right)
\end{array}\right\} .
\end{aligned}
$$

and using $X_{0}=0$ and $X_{1}=1$ in Proposition 2, we obtain the following result.

Proposition 3 (Cassini-like identity). For a natural numbers $n$, if $K_{n}^{(3)}$ is the $n$-th third-order Jacobsthal numbers, then the identity

$$
\begin{aligned}
& J_{n+1}^{(3)}(x) J_{n-1}^{(3)}(x)-\left(J_{n}^{(3)}(x)\right)^{2} \\
& =\frac{1}{\left(x^{2}+x+1\right)^{2}}\left\{\begin{array}{c}
x^{n}\left(\left(x^{2}-1\right) Z_{n+1}(x)+x(x+2) Z_{n}(x)\right) \\
-\left(x^{2}+x+1\right)
\end{array}\right\} .
\end{aligned}
$$

is true.

The d'Ocagne-like identity can also be obtained using the Binet formula and in this case we obtain

Proposition 4 (d'Ocagne-like identity). For a natural numbers $m$, $n$, with $m \geq n$ and $J_{n}^{(3)}(x)$ is the $n$-th third-order Jacobsthal polynomial, then the following identity

$$
\begin{aligned}
& J_{m+1}^{(3)}(x) J_{n}^{(3)}(x)-J_{m}^{(3)}(x) J_{n+1}^{(3)}(x) \\
& =\frac{1}{\left(x^{2}+x+1\right)^{2}}\left\{\begin{array}{c}
x^{m+1}\left(Z_{n+1}(x)-x Z_{n}(x)\right) \\
-x^{n+1}\left(Z_{m+1}(x)-x Z_{m}(x)\right)+\left(x^{2}+x+1\right) X_{m-n}
\end{array}\right\}
\end{aligned}
$$

is true.

Proof. Using the Eq. (2.4) and the Theorem 2, we get the required result.

In addition, some formulae involving sums of terms of the third-order Jacobsthal polynomial sequence will be provided in the following proposition. 
Proposition 5. For a natural numbers $m$, $n$, with $n \geq m$, if $J_{n}^{(3)}(x)$ and $K_{n}^{(3)}(x)$ are, respectively, the $n$-th third-order Jacobsthal and modified third-order Jacobsthal polynomials, then the following identities are true:

$$
\begin{gathered}
\sum_{s=m}^{n} J_{s}^{(3)}(x)=\frac{1}{3(x-1)}\left\{\begin{array}{c}
(3 x-2) J_{n}^{(3)}(x)+(2 x-1) J_{n-1}^{(3)}(x) \\
+x J_{n-2}^{(3)}(x)-J_{m+2}^{(3)}(x) \\
+(x-2) J_{m+1}^{(3)}(x)+(2 x-3) J_{m}^{(3)}(x)
\end{array}\right\}, \\
\sum_{s=0}^{n} K_{s}^{(3)}(x)=\frac{1}{x-1}\left\{\begin{array}{ccc}
x^{n+1}+2 x-3 & \text { if } & n \equiv 0(\bmod 3) \\
x^{n+1}+x-2 & \text { if } & n \equiv 1(\bmod 3) \\
x^{n+1}-1 & \text { if } & n \equiv 2(\bmod 3)
\end{array}\right.
\end{gathered}
$$

Proof. (3.1): Using Eq. (2.1), we obtain

$$
\begin{aligned}
\sum_{s=m}^{n} J_{s}^{(3)}(x)= & J_{m}^{(3)}(x)+J_{m+1}^{(3)}(x)+J_{m+2}^{(3)}(x)+\sum_{s=m+3}^{n} J_{s}^{(3)}(x) \\
= & J_{m}^{(3)}(x)+J_{m+1}^{(3)}(x)+J_{m+2}^{(3)}(x)+(x-1) \sum_{s=m+2}^{n-1} J_{s}^{(3)}(x) \\
& +(x-1) \sum_{s=m+1}^{n-2} J_{s}^{(3)}(x)+x \sum_{s=m}^{n-3} J_{s}^{(3)}(x)
\end{aligned}
$$

Then,

$$
\begin{aligned}
\sum_{s=m}^{n} J_{s}^{(3)}(x)= & (3 x-2) \sum_{s=m}^{n} J_{s}^{(3)}(x)+J_{m+2}^{(3)}(x)-(x-2) J_{m+1}^{(3)}(x)-(2 x-3) J_{m}^{(3)}(x) \\
& -(3 x-2) J_{n}^{(3)}(x)-(2 x-1) J_{n-1}^{(3)}(x)-x J_{n-2}^{(3)}(x) .
\end{aligned}
$$

Finally, the result in Eq. (3.1) is completed.

(3.2): As a consequence of the Eq. (2.4) of Theorem 2 and

$$
\begin{aligned}
\sum_{s=0}^{n} Y_{s} & =\sum_{s=0}^{n}\left(\omega_{1}^{s}+\omega_{2}^{s}\right) \\
& =\frac{\omega_{1}^{n+1}-1}{\omega_{1}-1}+\frac{\omega_{2}^{n+1}-1}{\omega_{2}-1} \\
& =\frac{1}{3}\left(Y_{n}-Y_{n+1}\right)+1,
\end{aligned}
$$

we have

$$
\begin{aligned}
\sum_{s=0}^{n} K_{s}^{(3)}(x) & =\sum_{s=0}^{n} x^{s}+\sum_{s=0}^{n} Y_{s} \\
& =\frac{x^{n+1}-1}{x-1}+\frac{1}{3}\left(Y_{n}-Y_{n+1}\right)+1
\end{aligned}
$$




$$
=\frac{1}{x-1}\left\{\begin{array}{cl}
x^{n+1}+2 x-3 & \text { if } \\
x^{n+1}+x-2 & \text { if } n \equiv 1(\bmod 3) \\
x^{n+1}-1 & \text { if } n \equiv 2(\bmod 3)
\end{array} .\right.
$$

Hence, we obtain the result.

For example, if $n \equiv 0(\bmod 3)$ we have that $x^{n+1}+2 x-3$ is divisible by $x-1$.

For negative subscripts terms of the sequence of modified third-order Jacobsthal polynomial we can establish the following result:

Proposition 6. For a natural number $n$ and $x^{3} \neq 0$ the following identities are true:

$$
\begin{aligned}
K_{-n}^{(3)}(x) & =K_{n}^{(3)}(x)+x^{-n}-x^{n}, \\
\sum_{s=0}^{3 n} K_{-s}^{(3)}(x) & =\frac{1}{x-1}\left(3 x-2-x^{-3 n}\right) .
\end{aligned}
$$

Proof. (3.3): Since $Y_{-n}=Y_{n}$, using the Binet formula stated in Theorem 2 and the fact that $\omega_{1} \omega_{2}=1$, all the results of this Proposition follow. In fact,

$$
\begin{aligned}
K_{-n}^{(3)}(x) & =x^{-n}+Y_{-n} \\
& =x^{-n}+x^{n}+Y_{n}-x^{n} \\
& =K_{n}^{(3)}(x)+x^{-n}-x^{n} .
\end{aligned}
$$

So, the proof is completed.

(3.4): The proof is similar to the proof of Eq. (3.1) using Eq. (3.3).

\section{CONCLUSION}

Sequences of polynomials have been studied over several years, including the well-known Tribonacci polynomial and, consequently, on the Tribonacci-Lucas polynomial. In this paper, we have also contributed for the study of third-order Jacobsthal and modified third-order Jacobsthal polynomials, deducing some formulae for the sums of such polynomials, presenting the generating functions and their Binet-style formula. It is our intention to continue the study of this type of sequences, exploring some their applications in the science domain. For example, a new type of sequences in the quaternion algebra with the use of these polynomials and their combinatorial properties.

\section{ACKNOWLEDGEMENTS}

The author also thanks the suggestions sent by the reviewer, which have improved the final version of this article. 


\section{REFERENCES}

[1] P. Barry, "Triangle geometry and Jacobsthal numbers," Irish Math. Soc. Bulletin, vol. 51, no. 1, pp. 45-57, 2003.

[2] G. Cerda-Morales, "Identities for third order Jacobsthal quaternions," Adv. Appl. Clifford Algebr., vol. 27, no. 2, pp. 1043-1053, 2017, doi: 10.1007/s00006-016-0654-1.

[3] G. Cerda-Morales, "Dual third-order Jacobsthal quaternions," Proyecciones Journal of Mathematics, vol. 37, no. 4, pp. 731-747, 2018.

[4] G. Cerda-Morales, "On the third-order Jacobsthal and third-order Jacobsthal-Lucas sequences and their matrix representations," Mediterr. J. Math., vol. 16, no. 2, pp. 1-12, 2019, doi: 10.1007/s00009-019-1319-9.

[5] C. K. Cook and M. R. Bacon, "Some identities for Jacobsthal and Jacobsthal-Lucas numbers satisfying higher order recurrence relations," Ann. Math. Inform, vol. 41, no. 1, pp. 27-39, 2013.

[6] G. B. Djordjević and H. M. Srivastava, "Incomplete generalized Jacobsthal and JacobsthalLucas numbers," Mathl. Comput. Modelling, vol. 42, pp. 1049-1056, 2005, doi: 10.1016/j.mcm.2004.10.026.

[7] G. B. Djordjević and H. M. Srivastava, "Some generalizations of the incomplete Fibonacci and the incomplete Lucas polynomials," Adv. Stud. Contemp. Math., vol. 11, pp. 11-32, 2005.

[8] G. B. Djordjević and H. M. Srivastava, "Some generalizations of certain sequences associated with the Fibonacci numbers," J. Indonesian Math. Soc., vol. 12, pp. 99-112, 2006.

[9] A. F.Horadam, "Jacobsthal representation numbers," Fibonacci Q., vol. 34, no. 1, pp. 40-54, 1996.

[10] R. K. Raina and H. M. Srivastava, "A class of numbers associated with the Lucas numbers," Mathl. Comput. Modelling, vol. 25, no. 7, pp. 15-22, 1997, doi: 10.1016/S0895-7177(97)00045-9.

Author's address

Gamaliel Cerda-Morales

Instituto de Matemáticas, Pontificia Universidad Católica de Valparaíso, Blanco Viel 596, Valparaíso, Chile

E-mail address: gamaliel.cerda.m@mail.pucv .cl 